Rev. Elev. Méd. vét. Pays trop., 1973, 26 (1) : 89-92

\title{
Contrôles trichinoscopiques des viandes de phacochères au Sénégal
}

\author{
par G. VASSILIADES $\left({ }^{*}\right)$
}

\begin{abstract}
RESUME
Après 440 analyses trichinoscopiques de viandes de phacochères effectuées au laboratoire d'helminthologie, de 1967 à 1972, on peut estimer que le pourcentage d'infestation par Trichinella spiralis des phacochères sauvages du Sénégal est de l'ordre de 4 p. 100.
\end{abstract}

La trichinose a été diagnostiquée au Sénégal pour la première fois par GRETILLAT et VASSILIADES en 1967 (6) chez le phacochère (Phacochoerus aethiopicus) et le chacal (Canis aureus), dans la région du delta du fleuve Sénégal. Les recherches de ces auteurs avaient été orientées dans cette voie à la suite d'une petite épidémie en mai 1966, au cours de laquelle 9 Européens de Dakar furent atteints plus ou moins gravement de cette parasitose (ONDE et CARAYON, 1968) (10). Ces malades avaient consommé du jambon cru d'un phacochère en provenance de Boundoum.

Le Sénégal devenait ainsí le $3^{\mathbf{e}}$ pays africain au Sud du Sahara où la trichinose était mise en évidence, après le Kenya (FORRESTER, NELSON et SANDER, 1961) (2) et la Tanzanie (SACHS et TAYLOR, 1966) (11).

Dès lors, il devenait nécessaire de mettre en garde la population du Sénégal contre les risques de contamination de l'homme par consommation de viande de phacochère crue ou insuffisamment cuite, ce qui fut fait immédiatement par voie de presse, et d'organiser un contrôle systématique de la viande des phacochè-

(*) I.E.M.V.T., Laboratoire national de l'Elevage et de Recherches vétérinaires, B.P. 2057, Dakar-Hann, Sénégal. res abattus par les chasseurs amateurs, très nombreux au Sénégal, et tous friands de jambon cru.

Le service d'helminthologie du Laboratoire national de l'Elevage et de Recherches vétérinaires de Dakar a pu, dès 1967, organiser une unité d'analyses trichinoscopiques qui fonctionne chaque année pendant la saison de chasse, de décembre à avril. A la fin de la saison 1971-1972, soit après 6 ans de contrôles, le nombre de phacochères examinés s'élève à 440 .

Nous pensons utile de présenter les résultats complets de toutes ces analyses.

\section{PRATIQUE DE L'EXAMEN TRICHINOSCOPIQUE}

L'examen trichinoscopique fait appel à l'utilisation de lames porte-objet spéciales ( Trichinae Counting Slides $")$. Ces lames sont constituées de 2 pièces : 1 lame support rectangulaire, en verre épais $(23 \mathrm{~cm} \times 5 \mathrm{~cm} \times 0,7 \mathrm{~cm})$, divisée en 28 cases $(2,5 \mathrm{~cm} \times 1 \mathrm{~cm})$, et 1 lame couvercle de mêmes dimensions, que l'on peut serrer sur la lame support par un système de 2 vis de serrage.

A partir de l'échantillon de viande à analyser, dont le poids minimal a été fixé à $300 \mathrm{~g}$, 
sont effectués des prélèvements musculaires par grattage au bistouri, en plusieurs points de l'échantillon à contrôler. Après hachage, les prélèvements sont répartis sur la lame support jusqu'à la recouvrir complètement, puis étalés par compression après vissage de la lame supérieure sur la lame support.

Si la viande est salée (jambon cru en cours de maturation), on ajoute un peu d'eau aux fragments prélevés pour les réhydrater.

La lame porte-objet absorbe environ $3 \mathrm{~g}$ de viande hachée. Pour chaque échantillon à analyser, au moins 6 lames trichinoscopiques sont examinées à la loupe binoculaire, à un fort grossissement (objectifs 25 ou 50).

Les éléments parasitaires recherchés sont les kystes trichiniens renfermant des formes larvaires infestantes de Trichinella spiralis Owen, 1835. Ces kystes ont généralement la forme d'une navette ou d'un citron et mesurent $450 \mu$ à $800 \mu$ de long sur 200 à $250 \mu$ de large; leur paroi est mince et transparente et les pôles sont souvent coiffés par quelques petits globules graisseux. A l'intérieur, la larve, fortement enroulée sur elle-même, occupe la région centrale du kyste; elle mesure $860 \mu$ à $1 \mathrm{~mm}$ de long sur 22 à $30 \mu$ de large (GRETILLAT et VASSILIADES, 1967) (6).

Cependant, il convient d'attirer l'attention sur le fait qu'un processus de calcification plus ou moins avancé peut rendre le diagnostic plus difficile. En effet, une fois calcifié, le kyste devient opaque, plus ou moins ovalaire ou sans forme caractéristique, granuleux, de couleur jaune brunâtre à marron foncé; à l'intérieur, il est parfois difficile de reconnaître la larve réduite à un petit filament noir, parfois fragmenté.

Néanmoins, la présence d'un seul de ces kystes, comme dans le cas où les kystes observés sont encore en bon état, suffit à considérer la viande comme trichinée et à la déclarer impropre à la consommation.

Dans la mesure du possible, et notamment dans les infestations importantes, le nombre de kystes par gramme de viande analysée est compté pour apprécier le degré de l'infestation. Chez le phacochère, les chiffres varient de 1 à 57 kystes au gramme, suivant l'animal et la région anatomique considérée.

\section{RESULTATS}

Tous les résultats, par année, de 1967 à 1972, sont donnés dans les tableaux I et II.

\section{COMMENTAIRES}

Il ressort de l'analyse des résultats portant sur 6 années de trichinoscopie, que la trichinose du phacochère est une zoonose parfaitement établie au Sénégal avec un pourcentage d'infestation de l'ordre de 4 p. 100 , ce qui, du point de vue épidémiologique, peut être considéré comme une infestation assez importante pour constituer un danger permanent.

En effet, dans la plupart des pays européens où la trichinose est considérée comme endémique, les taux d'infestations du porc, principale source de contamination en Europe et en Amérique du Nord, sont moins élevés qu'au Sénégal. Par contre, aux U.S.A., les taux d'infestation sont nettement plus forts. Voici à titre d'exemples quelques chiffres indiqués par EUZEBY (1961) (1): «en Allemagne (1954): 0,003 p. 1.000 ; en Norvège : 0,02 p. 1.000 ; en Pologne, en Roumanie et en Grèce : 0,2 à 0,6 p. 1.000. Aux U.S.A. : 1 à 2 p. $100 \mathrm{chez}$ les animaux recevant une nourriture bien cuite; 20 p. 100 chez ceux nourris de résidus d'abattoirs ou de déchets alimentaires non cuits $\gg$.

Si l'on considère la répartition géographique des cas positifs au Sénégal, on pourrait être tenté de localiser cette affection à la région du Fleuve. En fait, cela tient probablement à ce que la région du Fleuve est, au Sénégal, la zone de prédilection des chasseurs qui trouvent dans cette région abondance de gibier et un terrain de chasse favorable à la réalisation de safaris au phacochère. Il est donc normal que la plupart des phacochères analysés proviennent de cette région et partant, que le nombre de cas positifs y soit le plus élevé.

Les recherches sur l'épidémiologie de la trichinose au Sénégal et sur les caractères biologiques de la souche ouest-africaine de Trichinella spiralis ont donné lieu à de nombreuses études [GRETILLAT et VASSILIADES, 1968 a (7), b (8), c (9)], [GRETILLAT et CHEVALLIER, 1969 (4), 1970 (5)], [GRETILLAT, 1971 (3)] d'où il ressort principalement que le réservoir naturel de cette anthropozoonose est constitué par les carnivores sauvages 
TABLEAU N $\mathrm{N}^{\circ}$

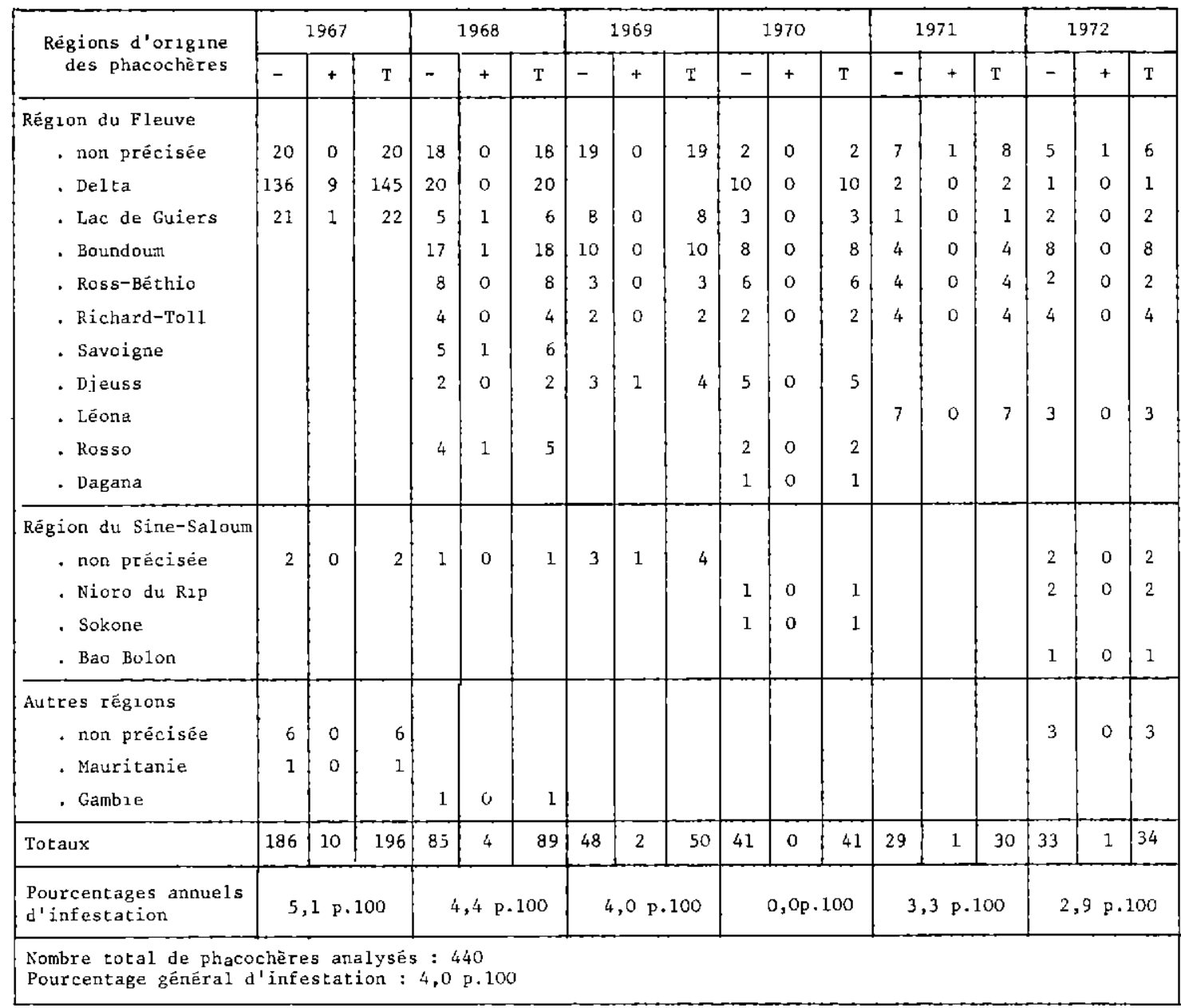

(- : nombre de phacochères à trichinoscopie négative; + i nombre de phacochères à trichinoscopic positive; $T$ : nombre total de phacochères examinés).

TABLEAU $\mathrm{N}^{\circ} \mathrm{II}$

Rẻcapitulation

\begin{tabular}{|c|c|c|c|c|}
\hline Annêes & Cas négatifs & Cas positifs & T o t a u $x$ & $\begin{array}{l}\text { Pourcentages } \\
\text { d'infestations }\end{array}$ \\
\hline 1967 & 186 & 10 & 196 & $5,1 \mathrm{p}, 100$ \\
\hline 1968 & 85 & 4 & 89 & 4,4 \\
\hline 2969 & 48 & 2 & 50 & 4,0 \\
\hline 1970 & 41 & 0 & 41 & 0,0 \\
\hline 1971 & 29 & 1 & 30 & 3,3 \\
\hline 1972 & 33 & 1 & 34 & 2,9 \\
\hline Totaux & 422 & 18 & 440 & 4,0 \\
\hline
\end{tabular}

chez qui la souche est particulièrement bien adaptée. Le phacochère ne peut être considéré que comme un hôte accidentel, de même que l'homme. Il s'agit d'un cycle naturel sylvatique différent du schéma épidémiologique classique basé sur la transmission de la trichinose par le porc domestique.

Cependant, bien que l'infestation naturelle du porc domestique n'ait pas encore été constatée au Sénégal, il a été possible, en labora- 
toire, de réaliser expérimentalement l'infestation du porc par une souche sénégalaise de Trichinella spiralis. Aussi la trichinose des animaux sauvages, au Sénégal, doit-elle être considérée comme un danger latent devant les risques de dispersion de cette zoonose, dans les élevages de porcs.

Pour ce qui concerne la trichinose humaine, dont on sait qu'elle est une des affections à nématodes les plus graves chez l'homme, puisque souvent mortelle, l'infestation naturelle du phacochère représente un danger certain du fait de la consommation de viande crue (" jam- bon de Bayonne 》) ou insuffisamment cuite. Fort heureusement, la population du Sénégal, à majorité musulmane, par ses habitudes culinaires et des interdits religieux est à l'abri de tels accidents (cuisson prolongée des viandes, interdiction de consommer du porc et autres suidés sauvages, etc.).

Par contre, les amateurs de safaris, pour la plupart des Européens résidant au Sénégal ou touristes de passage, doivent être mis en garde et protégés contre cette affection qui, si elle est relativement rare, n'en demeure pas moins d'une extrême gravité.

\section{SUMMARY}

\section{Survey on trichinosis of wart-hogs in Senegal by trichinoscopy method}

In Senegal, from 1967 to 1972, 440 wart-hogs were examinated by trichinoscopy method. 4 p. 100 of them were found to be infested with Trichinella spiralis.

\section{RESUMEN}

\section{Controles triquinoscopicos de las carnes de facoquerios en Senegal}

Después de 440 analisis triquinoscopicos de carnes de facoquerios efectuados en el laboratorio de helmintologia de Dakar, de 1967 a 1972 , se puede estimar que el porcentaje de infestación por Trichinella spiralis de los facoquerios salvajes de Senegal es de unos 4 p. 100.

\section{BIBLIOGRAPHIE}

1. EUZEBY (J.). Les maladies vermineuses des animaux domestiques et leurs incidences sur la pathologie humaine. T. 1, fasc. I, 1961, 473 p., Paris, Vigot Frères.

2. FORRESTER (A.T.T.), NELSON (G.S.) et SANDER (G.). The first record of an outbreak of trichinosis in Africa south of the Sahara, Trans. Roy. Soc. Trop. Med. Hyg., 1961, 55 (6) : 503-513.

3. GRETILLAT (S.). La trichinose des animaux sauvages en Afrique doit être considérée comme une zoonose d'avenir. Econ. Méd. anim., 1971 , 12 (2) : 113-116.

4. GRETILLAT (S.) et CHEVALIER (J. L.). Réceptivité du phacochère (Phacochoerus aethiopicus) à la souche ouest-africaine de Trichinella spiralis. C.R. Acad. Sci. Paris, 1969, 269 : 2381-2383.

5. GRETILLAT (S.) et CHEVALIER (J. L.). Note préliminaire sur l'épidémiologie de la trichinose des animaux sauvages en Afrique de l'Ouest. Bull. Org. mond. santé, 1970, 43: 749-757.

6. GRETILLAT (S.) et VASSILIADES (G.). Présence de Trichinella spiralis (Owen, 1835) chez les carnivores et suidés sauvages de la région du
Delta du Fleuve Sénégal. C.R. Acad. Sci. Paris 1967, 264 : 1297-1300.

7. GRETILLAT (S.) et VASSILIADES (G.). Particularités biologiques de la souche ouest-africaine de Trichinella spiralis (Owen, 1835). Réceptivité et sensibilité de quelques mammifères domestiques et sauvages. Rev. Elev. Méd. vét. Pays trop., $1968: 21$ (1) : 85-99.

8. GRETILLAT (S.) et VASSILIADES (G.). Réceptivités comparées du chat et du porc domestiques à la souche ouest-africaine de Trichinella spiralis. C.R. Acad. Sci. Paris, 1968 b, 266 : 11391141.

9. GRETILLAT (S.) et VASSILIADES (G.). La trichinose expérimentale du singe (souche ouestafricaine de Trichinella spiralis (Owen, 1835). Bull. Soc. Path. exot., 1968 c, 61 (2): 246-251.

10. ONDE (M.) et CARAYON (A.). Cas dakarois de trichinose. Bull. Soc. Méd. Afr. Noire Lgue fr., 1968,13 (2) : 332-336.

11. SACHS (R.) et TAYLOR (A.S.). Trichinosis in a spotted Hyaena (Crocuta crocuta) of the Serengeti. Vet. Rec., 1966, 78 : 704. 